\title{
Real-time Warning and Signal Verification for Infectious Disease Outbreaks using Syndromic Surveillance System in Rural Jiangxi Province, China
}

\author{
Tao Tao*1, Changming Zhou ${ }^{1}$, Xiaoxiao Song ${ }^{1}$, Qi Zhao ${ }^{1}$, Per Andersson², Weirong Yan ${ }^{3,4}$ \\ and Biao $\mathrm{Xu}^{1}$
}

${ }^{1}$ School of Public Health, Fudan University, Shanghai, China; ${ }^{2}$ Invotech Solutions, Gävle, Sweden; ${ }^{3}$ Division of Global Health (IHCAR), Department of Public Health Sciences, Karolinska Institutet, Stockholm, Sweden; ${ }^{4}$ Department of Epidemiology and Biostatistics, School of Public Health, Tongji Medical College, Huazhong University of Science and Technology, Wuhan, China

\section{Objective}

Our aim was to explore the practical performance of ISSC system through investigating the characteristics and verifying results of alert signals raised during real-time surveillance periods.

\section{Introduction}

Despite the global emergence of syndromic surveillance systems and theories in recent years, the practical performance of this surveillance method under real circumstances had been rarely evaluated, especially in resource-limited areas. Since April 2012, a syndromic surveillance system named "ISSC" has been established among health care facilities, pharmacies and primary schools in two rural counties (County A \& B) of Jiangxi Province, China.

\section{Methods}

Daily syndromic data sources, which included main complaints and preliminary diagnosis of outpatients from health care facilities, drug sales from pharmacies and school absenteeism reports from primary schools, were collected and transferred to a web-based electronic surveillance system (ISSC system). A series of alarm triggers targeting different data sources in each surveillance unit were set in ISSC system, which would automatically run at 12:00 every day to detect potential aberrations among syndromic data reported one day before (for primary schools, the alarm trigger targeted the same day's data). Owing to the unknown baseline feature and outbreak manifestation, simple algorithms including Standard (fixed value as threshold) and Shewhart Chart (mean plus 3 deviations as threshold, 7 days as baseline) were introduced in the alarm triggers. Once an alarm was raised by the alarm trigger, two epidemiological researchers from Fudan University would manually check the data records in ISSC system for initial verification. If any suspect syndrome or disease cluster was found in the automatic alarms, a formal "alert signal" would be sent by email to the disease control staffs in local CDCs for further investigation.

\section{Results}

From March 18th to August 17th, 2013, totally 46 suspect signals were sent to local CDCs for investigation. Most of the signals (71.7\%) came from County A, and health care facility and primary school were the main sources of alert signals $(93.5 \%)$. Respiratory syndrome $(60.9 \%)$ and varicella $(17.4 \%)$ were the most frequent causes of alerting. Among these 46 alert signals, 2 influenza outbreaks (New-H1N1) and 1 hand-foot-mouth disease outbreak (CoxA16) were confirmed after investigations, and timely interventions were given by CDC staffs. Detailed characteristics were shown in Figure 1. According to the archives from local CDCs, totally 4 outbreaks occurred during the real-time surveillance period. Assuming no outbreak was missed in the study sites, ISSC warning system reached a sensitivity of $75.0 \%$ (3 outbreak detected / 4 outbreak occurred) and a specificity of $74.5 \%$
(114 non-alerting days / 153 total surveillance days). The positive predictive value (PPV) and negative predictive value (NPV) of outbreak detection were $6.5 \%$ and $93.5 \%$ respectively. None of the ISSC alert signals was correspondingly found from the China Information System for Disease Control and Prevention (CISDCP), which is a traditional case report system established since 2004 in study sites, before the confirmation of field alert investigation.

\section{Conclusions}

Combined with real-time warning and epidemiological verification, the ISSC syndromic surveillance system has the ability to detect infectious disease outbreaks which might be missed by traditional case report system. The number of false positive alert signals would be further decreased by incorporating advanced detection algorithms and exploring the manifestations of disease outbreak in rural community.

\begin{tabular}{lllll}
\multicolumn{5}{c}{ Table 1 Characteristics of waming signals from March $18^{\text {th }}$ to August $17^{\text {th }}, 2013$} \\
\hline Item & & County A & County B & Total \\
\hline All signals & 33 & 13 & 46 \\
Source of signal & Health care facility & 18 & 9 & 27 \\
& Pharmacy & 2 & 1 & 3 \\
& Primary school & 13 & 3 & 16 \\
Category of signal & Respiratory & 20 & 8 & 28 \\
& Syndrome & 2 & 1 & 3 \\
& Gastroirtestinal & 2 & 1 & 1 \\
& Syndrome & 0 & 2 & 8 \\
& Rash Syndrome & 0 & 0 & 2 \\
& Varicella & 6 & 0 & 1 \\
& Mumps & 2 & 1 & 3 \\
& Rubella & 1 & 0 & 3 \\
& Surge of Drug Sale & 2 & 11 & 39 \\
Investigationresult & Outbreak & 3 & 2 & 4 \\
& Non-outbreak & 28 & 2 &
\end{tabular}

* Caused by the misoperation of data reporters

\section{Keywords}

rea-time warning; alert signal; syndromic surveillance

\section{Acknowledgments}

This study was funded by European Union's Seventh Framework Programmer ([FP7/2007-2013] [FP7/2007-2011]) under grant agreement no. [241900].

\section{*Tao Tao}

E-mail: ttsuper2000@hotmail.com 